Supplementary Information for

\title{
Mechanistic and thermodynamic insights into anion exchange by green rust
}

By Myriam I. Agnel*, Sylvain Grangeon, François Fauth, Erik Elkaïm, Francis Claret, Marjorie Roulet, Fabienne Warmont, Christophe Tournassat

Myriam I. Agnel

Email: myriam.agnel@cnrs-orleans.fr

\section{Contents:}

Supplementary text

Figs. S1 to S7

Tables S1 to S4

References for SI reference citations 


\section{Supplementary Information Text}

\section{Scherrer equation}

Scherrer equation is: ${ }^{1}$

$$
D=\frac{k \lambda}{F W H M \cos (\theta)}
$$

in which D is the crystallite size $(\AA)$, $\mathrm{k}$ is a shape constant (equal to 1$), \lambda$ is the $\mathrm{X}$-ray wavelength ( $)$, FWHM is the full width at half-maximum of the diffraction peak (rad) and $\theta$ is the Bragg angle (rad).

\section{Calculation of XRD patterns (Fig. S6)}

XRD patterns were calculated using micro-GR(Cl) as a starting structure model (Fig. S6, black line: micro-GR(Cl)) and working with the software CALCIPOW. ${ }^{2}$ Mean and maximum number of layers were set to 12 and 15 respectively (lognormal distribution). Radius of crystallite in the ab plane was fixed to $50 \AA$. The amount of stacking defects were set to: $0 \%$ - 3D-ordered structure (micro-GR(Cl) in this case, black line on Fig. S6), $50 \%$ (light blue line on Fig. S6A) and $100 \%$ turbostratism (red line on Fig. S6). The model from micro-GR(Cl) was also modified to obtain a green rust with two coherently stacked sheets on average (purple line on Fig. S6B). 


\section{Supplementary Figures}
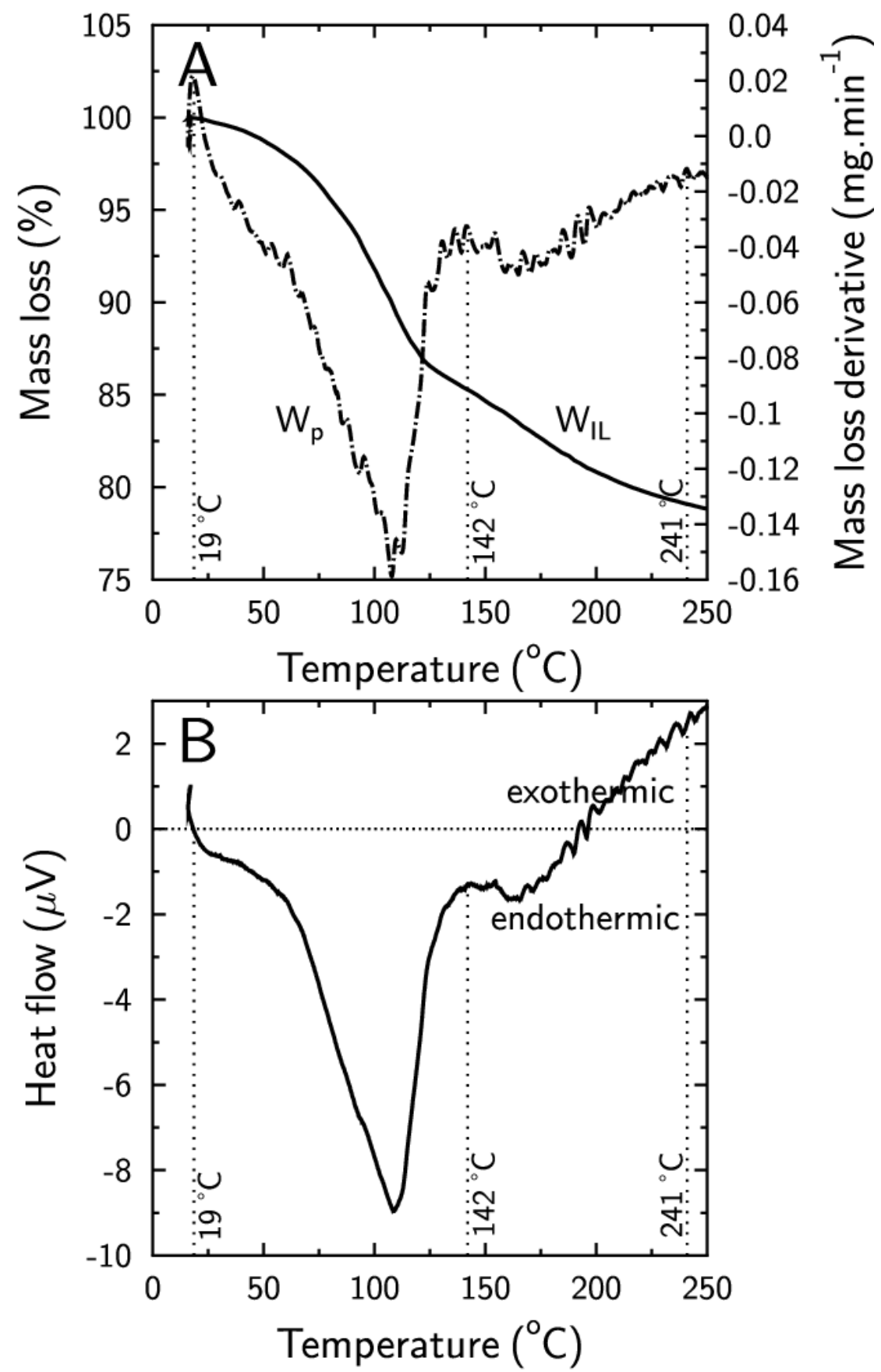

Fig. S1. A: TGA results for micro-GR(Cl). Full line: mass loss (\%); Dash line: mass loss derivative $\left(\mathrm{mg} \cdot \mathrm{min}^{-1}\right) ; \mathrm{W}_{\mathrm{P}}=$ interparticle $\left(\right.$ capillary) pore water; $\mathrm{W}_{\mathrm{IL}}=$ interlayer water. $\mathrm{B}$ : $\mathrm{DSC}$ results. 

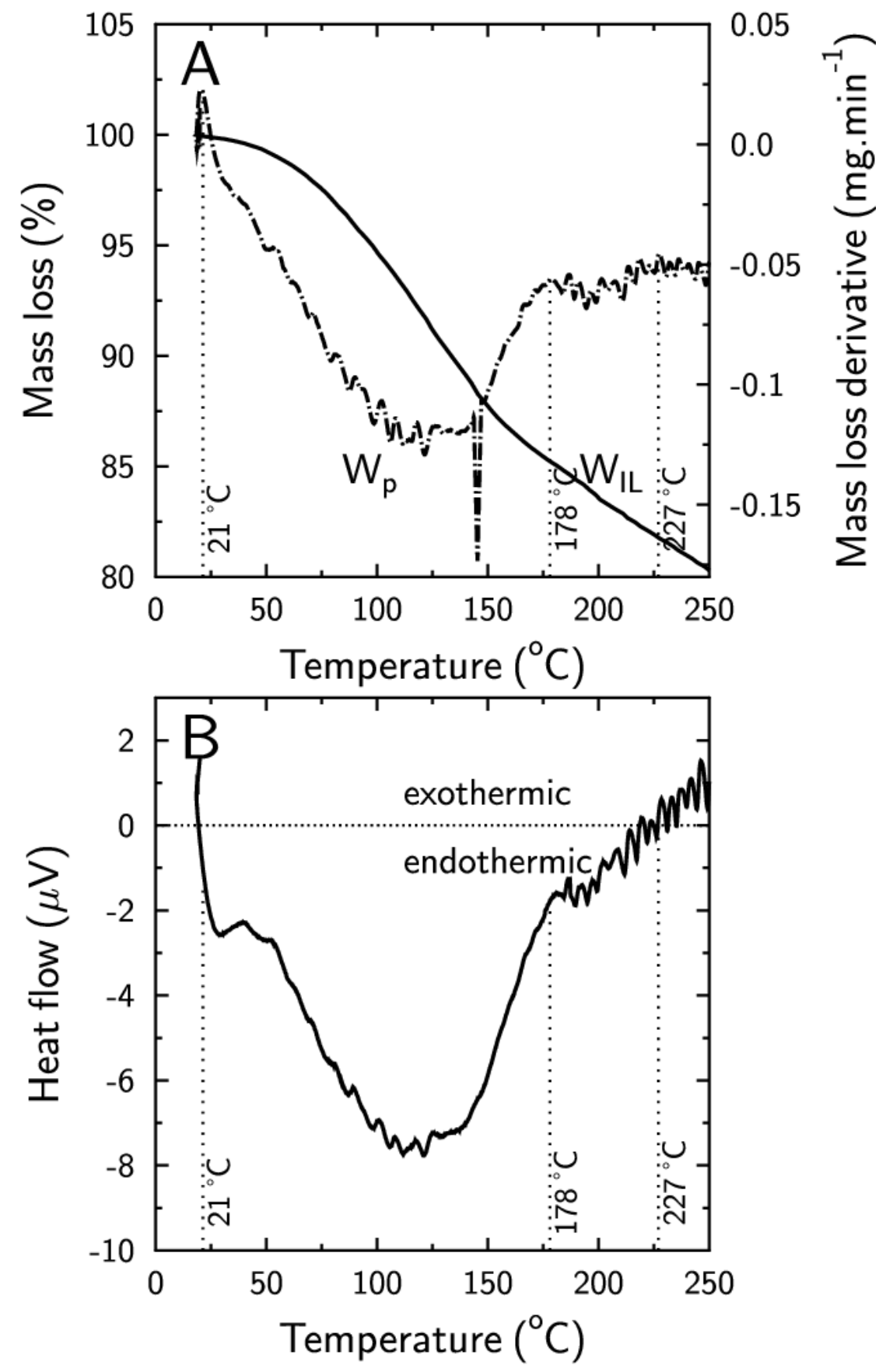

Fig. S2. A: TGA results for nano-GR(SO $\mathrm{SO}_{4}$. Full line: mass loss (\%); Dash line: mass loss derivative $\left(\mathrm{mg} \cdot \mathrm{min}^{-1}\right) ; \mathrm{W}_{\mathrm{P}}=$ interparticle (capillary) pore water; $\mathrm{W}_{\mathrm{IL}}=$ interlayer water. $\mathrm{B}$ : DSC results 

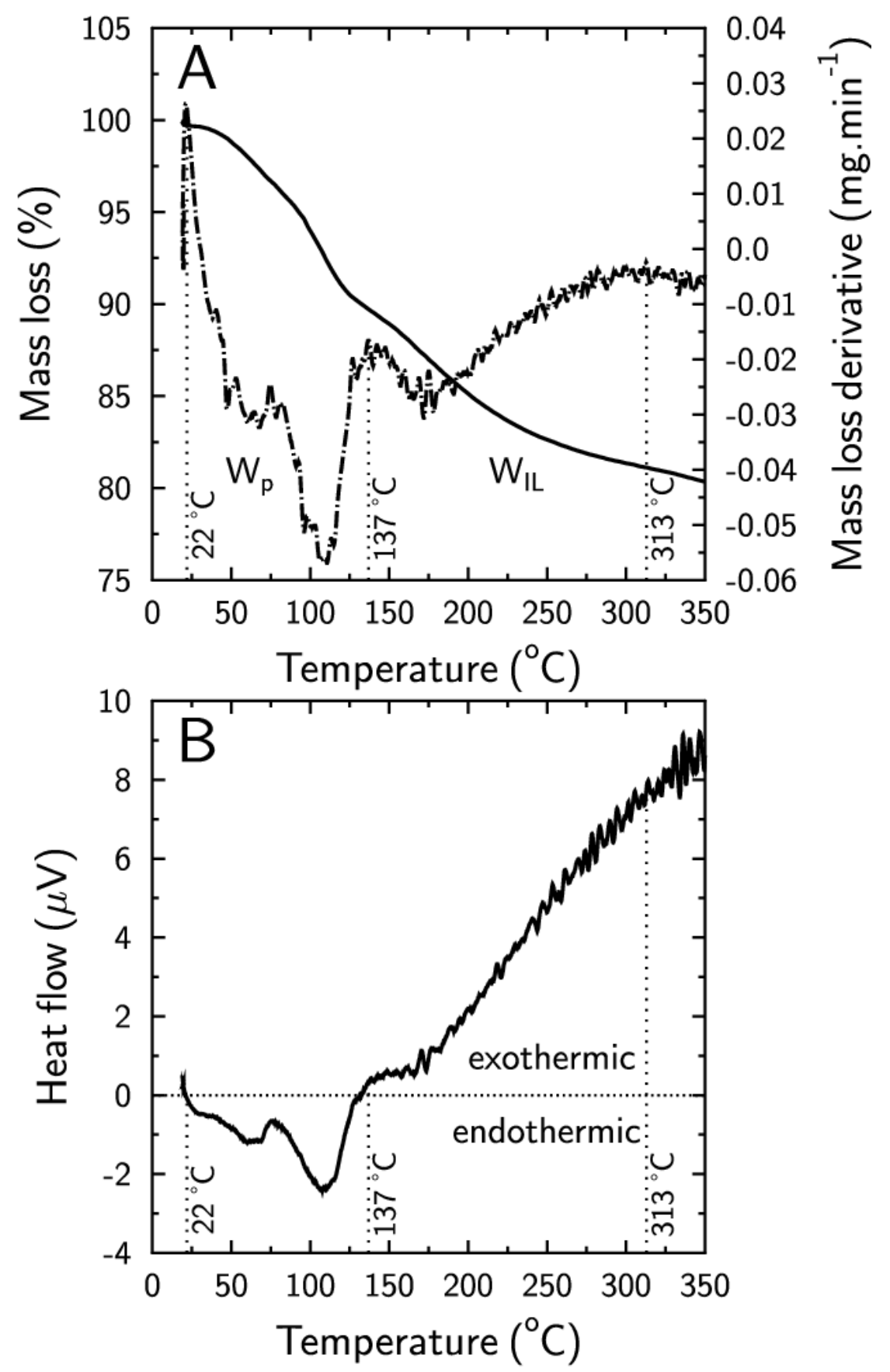

Fig. S3. A: TGA results for micro-GR( $\left(\mathrm{SO}_{4}\right)$. Full line: mass loss (\%); Dash line: mass loss derivative $\left(\mathrm{mg} \cdot \mathrm{min}^{-1}\right) ; \mathrm{W}_{\mathrm{P}}=$ interparticle (capillary) pore water; $\mathrm{W}_{\mathrm{IL}}=$ interlayer water. $\mathrm{B}$ : DSC results 


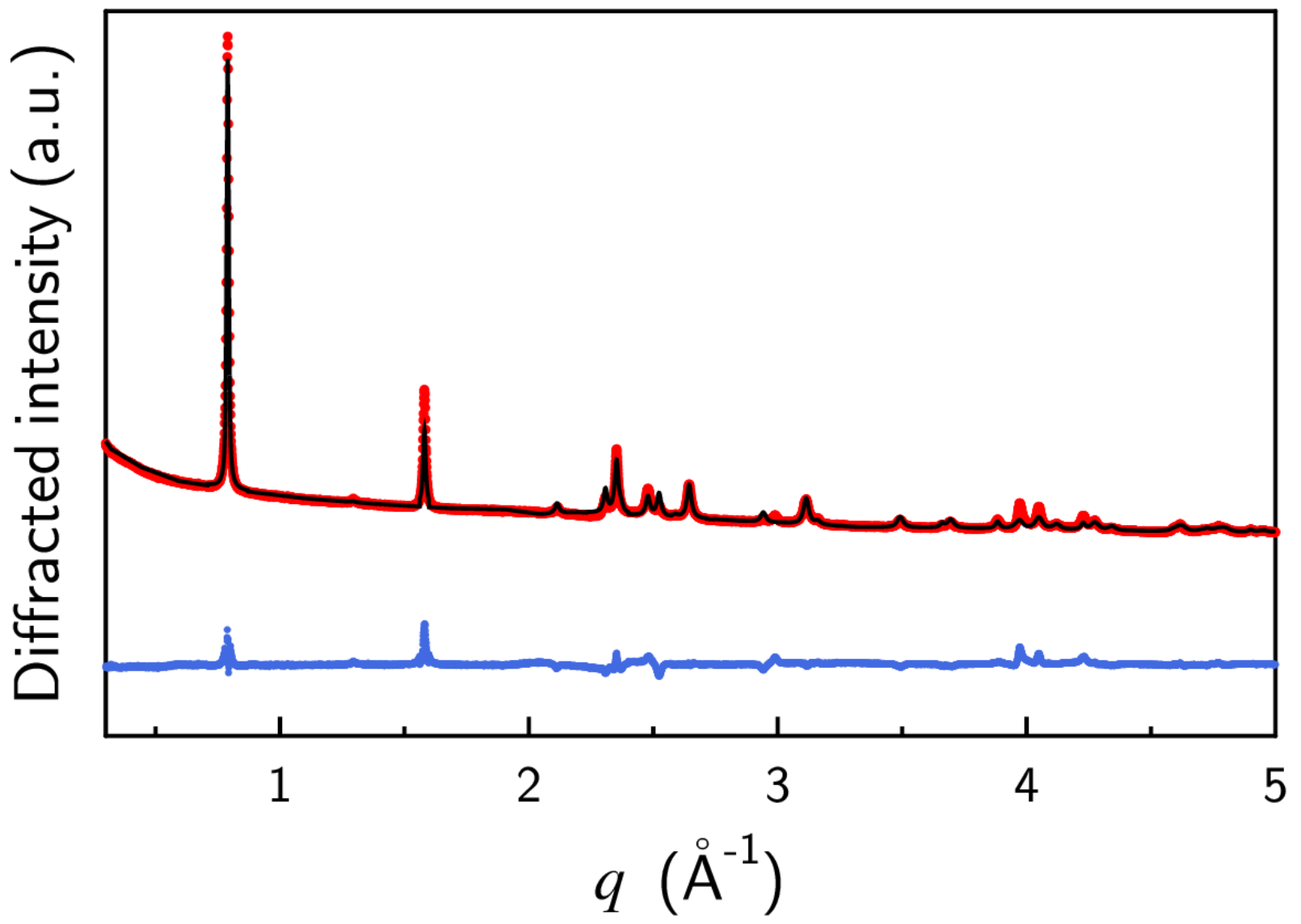

Fig. S4: Rietveld refinement results (black line) on micro-GR(Cl) XRD pattern (red line). Blue line is the residue between the data and the refinement results. 


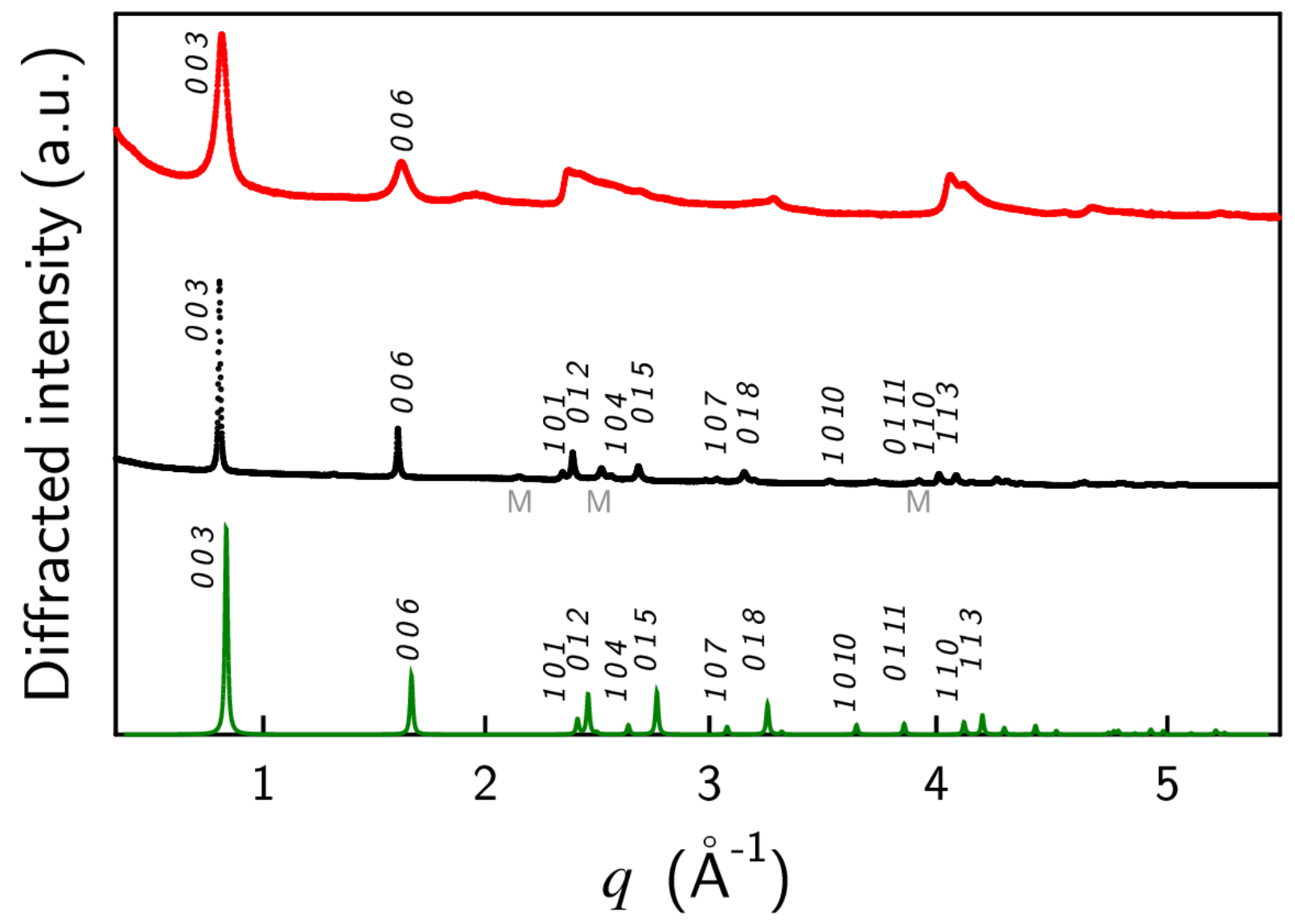

Fig. S5: XRD patterns of Fougerite ${ }^{3}$ (green line), micro-GR(Cl) (black line) and nano-GR(Cl) (red line). M: magnetite impurities in micro-GR( $\mathrm{Cl})$. Intensities were normalized to the maximum intensity of 003 reflection. 

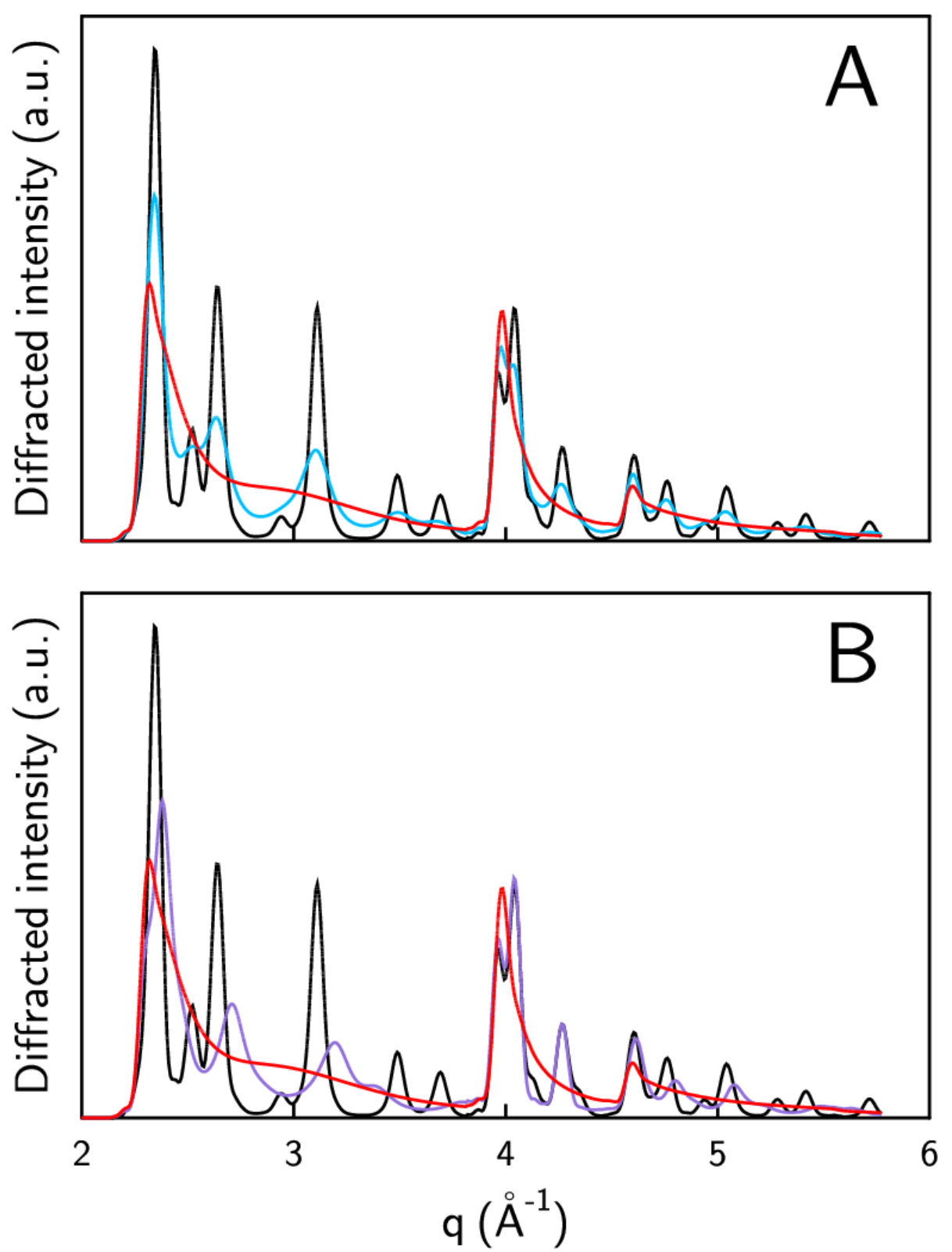

Fig. S6: Influence of stacking defects on micro-GR(Cl). Black line: initial micro-GR XRD pattern $-0 \%$ stacking defects $(\mathrm{A}$ and $\mathrm{B})$. Red line: $100 \%$ stacking defects $=$ turbostratism $(\mathrm{A}$ and $\mathrm{B})$. Light blue line: $50 \%$ stacking defects in the micro-GR(Cl) model (A). Purple line: two coherently stacked green rust sheets on average, assuming a lognormal distribution (B). 


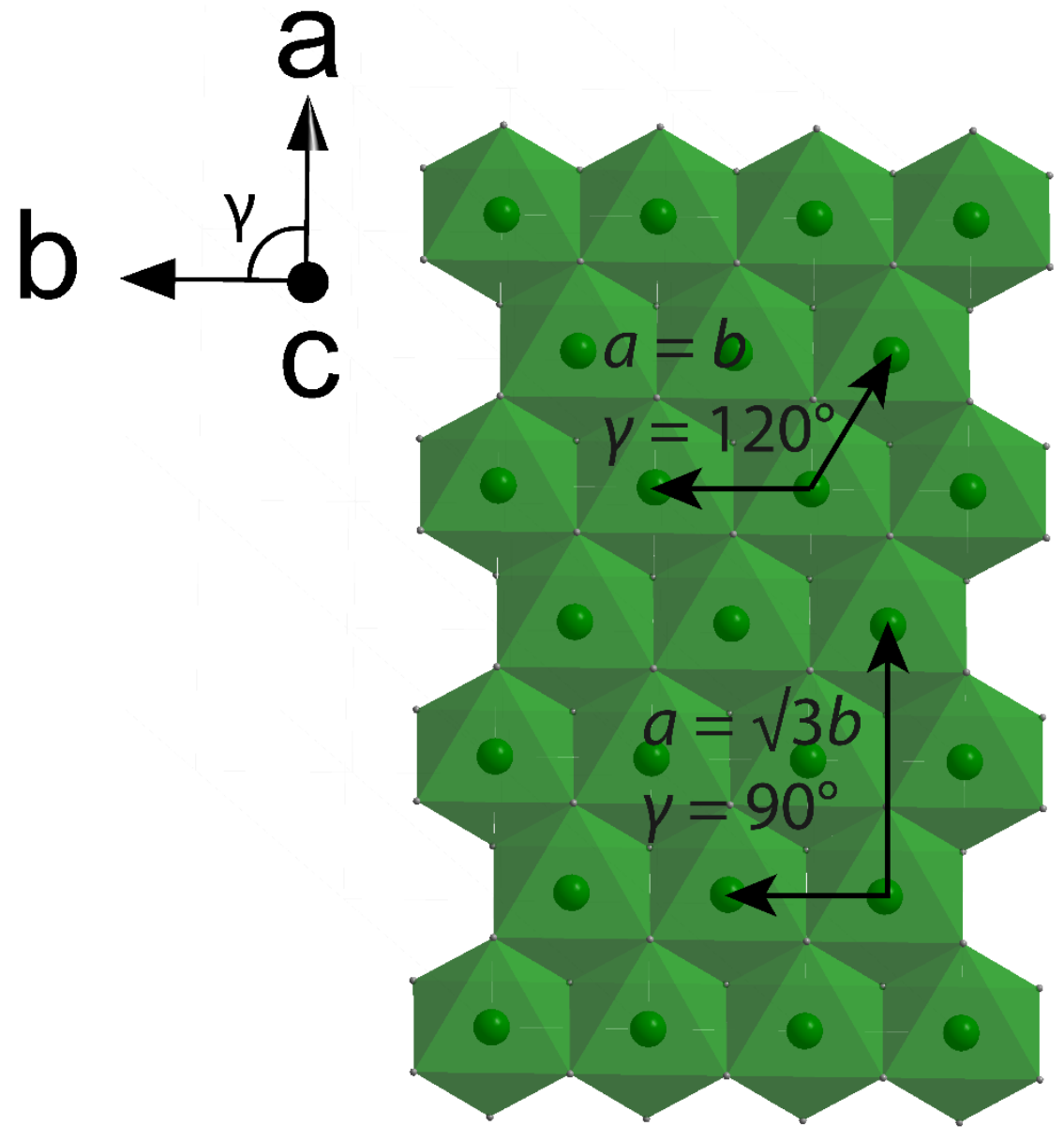

Fig. S7: Two equivalent hexagonal layer symmetries. If the layer symmetry is lowered, $a$ becomes different from $\sqrt{ } 3 \times b$ and the layer would not be hexagonal anymore. 
Table S1. Nomenclature and concentrations of solutions used for batch experiments

\begin{tabular}{lll}
\hline Name & $\begin{array}{l}\text { Concentration NaCl } \\
\left(\mathbf{m m o l}_{\mathbf{L}}^{-\mathbf{1}}\right)\end{array}$ & $\begin{array}{l}\text { Concentration Na2SO4 } \\
\left(\mathbf{m m o l}_{\mathbf{L}} \mathbf{- 1}\right)\end{array}$ \\
\hline C150 & 50 & 0 \\
S25 & 0 & 25 \\
S15 & 0 & 15 \\
S5 & 40 & 5 \\
S2.4 & 45 & 2.4 \\
S1 & 48 & 1 \\
S0.5 & 49 & 0.5 \\
\hline
\end{tabular}


Table S2. Protocol for the batch exchange experiments of micro-GR (see Table S1 for nomenclature of solutions, $\mathrm{AEC}=$ anionic exchange capacity, $\mathrm{K}_{\mathrm{ex}}=$ selectivity coefficient, $\mathrm{E}=$ Surface equivalent fraction)

\begin{tabular}{|c|c|c|c|c|c|}
\hline Protocol & Exp S25 & Exp S5 & Exp S2.4 & Exp S1 & Exp S0.5 \\
\hline $\begin{array}{c}\text { Equilibrium } \\
\text { phase }\end{array}$ & \multicolumn{5}{|c|}{$200 \mathrm{~mL} \mathrm{Cl50}$} \\
\hline Rinsing 1 & \multicolumn{5}{|c|}{$\begin{array}{c}\text { 30-40 mL Milli-Q Water } \\
\text { Direct Filtration }\end{array}$} \\
\hline $\begin{array}{l}\text { Exchange } \\
\text { phase } 1 \text { - } 1\end{array}$ & $200 \mathrm{~mL} \mathrm{~S} 25$ & $200 \mathrm{~mL} \mathrm{S5}$ & $20 \mathrm{~mL} \mathrm{~S} 2.4$ & $200 \mathrm{~mL} \mathrm{S1}$ & $200 \mathrm{~mL} \mathrm{~S} 0.5$ \\
\hline 2 & $200 \mathrm{~mL} \mathrm{~S} 25$ & 200 mL S5 & $40 \mathrm{~mL} \mathrm{~S} 2.4$ & 200 mL S1 & $200 \mathrm{~mL} \mathrm{~S} 0.5$ \\
\hline 3 & $200 \mathrm{~mL} \mathrm{~S} 25$ & 200 mL S5 & 60 mL S2.4 & 200 mL S1 & $200 \mathrm{~mL} \mathrm{~S} 0.5$ \\
\hline 4 & $\begin{array}{l}\text { End of this } \\
\text { experiment }\end{array}$ & $200 \mathrm{~mL} \mathrm{S5}$ & 100 mL S2.4 & 200 mL S1 & $200 \mathrm{~mL} \mathrm{~S} 0.5$ \\
\hline 5 & & $200 \mathrm{~mL} \mathrm{S5}$ & 200 mL S2.4 & 200 mL S1 & $200 \mathrm{~mL} \mathrm{~S} 0.5$ \\
\hline 6 & & & 200 mL S2.4 & & \\
\hline Rinsing 2 & & \multicolumn{4}{|c|}{$\begin{array}{c}\text { 30-40 mL Milli-Q Water } \\
\text { Direct Filtration }\end{array}$} \\
\hline $\begin{array}{l}\text { Exchange } \\
\text { phase } 2-1\end{array}$ & & $200 \mathrm{~mL} \mathrm{~S} 25$ & $200 \mathrm{~mL} \mathrm{S15}$ & $200 \mathrm{~mL} \mathrm{~S} 25$ & $200 \mathrm{~mL} \mathrm{~S} 25$ \\
\hline 2 & & & $200 \mathrm{~mL}$ S15 & $200 \mathrm{~mL} \mathrm{~S} 25$ & \\
\hline $\begin{array}{c}\text { Obtained } \\
\text { parameters }\end{array}$ & $\begin{array}{c}\text { Exchange } \\
\text { stoichiometry + } \\
\text { AEC }\end{array}$ & \multicolumn{4}{|c|}{$\log K_{e x}+E$} \\
\hline
\end{tabular}


Table S3. Protocol for the batch exchange experiments of nano-GR (see Table S1 for nomenclature of solutions, $\mathrm{AEC}=$ anionic exchange capacity, $\mathrm{K}_{\mathrm{ex}}=$ selectivity coefficient, $\mathrm{E}=$ Surface equivalent fraction)

\begin{tabular}{|c|c|c|c|}
\hline Protocol & $\operatorname{Exp~S25~}$ & Exp S5 & $\operatorname{Exp~S2.4~}$ \\
\hline Equilibrium phase & \multicolumn{3}{|c|}{$200 \mathrm{~mL} \mathrm{Cl50}$} \\
\hline Rinsing 1 & & $\begin{array}{l}\text { aL Milli-Q W } \\
\text { ect Filtration }\end{array}$ & \\
\hline Exchange phase 1 - 1 & $200 \mathrm{~mL} \mathrm{~S} 25$ & $200 \mathrm{~mL} \mathrm{S5}$ & $10 \mathrm{~mL} \mathrm{~S} 2.4$ \\
\hline 2 & $200 \mathrm{~mL} \mathrm{~S} 25$ & $200 \mathrm{~mL} \mathrm{S5}$ & $10 \mathrm{~mL} \mathrm{~S} 2.4$ \\
\hline 3 & $200 \mathrm{~mL} \mathrm{~S} 25$ & $200 \mathrm{~mL} \mathrm{S5}$ & $10 \mathrm{~mL} \mathrm{~S} 2.4$ \\
\hline 4 & $\begin{array}{l}\text { End of this } \\
\text { experiment }\end{array}$ & $200 \mathrm{~mL} \mathrm{S5}$ & $15 \mathrm{~mL} \mathrm{~S} 2.4$ \\
\hline 5 & & $200 \mathrm{~mL} \mathrm{S5}$ & $20 \mathrm{~mL} \mathrm{~S} 2.4$ \\
\hline 6 & & & $20 \mathrm{~mL} \mathrm{~S} 2.4$ \\
\hline Rinsing 2 & & $\begin{array}{r}30-40 \mathrm{~m} \\
\text { Dir }\end{array}$ & $\begin{array}{l}\text { Q Water } \\
\text { tion }\end{array}$ \\
\hline Exchange phase 2 - 1 & & $200 \mathrm{~mL} \mathrm{~S} 25$ & $200 \mathrm{~mL} \mathrm{S15}$ \\
\hline 2 & & & $200 \mathrm{~mL} \mathrm{S15}$ \\
\hline Obtained parameters & $\begin{array}{c}\text { Exchange } \\
\text { stoichiometry + AEC }\end{array}$ & & \\
\hline
\end{tabular}


Table S4. Stoichiometries of $\mathrm{Fe}, \mathrm{Co}, \mathrm{Cl}$ and $\mathrm{S}$ contents in studied samples determined by EPMA (on the basis of $\mathrm{Fe}+\mathrm{Co}=1$ ). Each number is an average on the number of independent measurements ( $\mathrm{n} ; \pm$ standard deviation). $\mathrm{Fe}(\mathrm{III})$ stoichiometry is inferred from $\mathrm{Cl}$ stoichiometry or $\mathrm{SO}_{4}$ stoichiometry times 2 and $\mathrm{Fe}(\mathrm{II})=\mathrm{Fe}-\mathrm{Fe}(\mathrm{III})$.

\begin{tabular}{|c|c|c|c|c|c|c|c|}
\hline Sample & $\mathbf{n}$ & $\mathbf{F e}$ & Co & $\mathrm{Fe}($ III) & $\mathrm{Fe}(\mathrm{II})$ & Cl & $\mathbf{S}$ \\
\hline Micro- & 61 & $0.67 \pm$ & $0.33 \pm$ & $0.25 \pm$ & $0.42 \pm$ & $0.25 \pm$ & $0 \pm 0$ \\
\hline GR(Cl) & & 0.04 & 0.04 & 0.04 & 0.08 & 0.04 & \\
\hline Micro- & 49 & $0.66 \pm$ & $0.34 \pm$ & $0.22 \pm$ & $0.44 \pm$ & $0 \pm 0$ & $0.11 \pm$ \\
\hline GR(SO4) & & 0.02 & 0.02 & 0.02 & 0.04 & & 0.01 \\
\hline Nano- & 35 & $0.49 \pm$ & $0.51 \pm$ & $0.2 \pm$ & $0.29 \pm$ & $0.2 \pm$ & $0 \pm 0$ \\
\hline GR(Cl) & & 0.05 & 0.05 & 0.03 & 0.06 & 0.03 & \\
\hline Nano- & 53 & $0.52 \pm$ & $0.48 \pm$ & $0.16 \pm$ & $0.37 \pm$ & $0 \pm 0$ & $0.08 \pm$ \\
\hline GR(SOH) & & 0.03 & 0.03 & 0.02 & 0.05 & & 0.01 \\
\hline
\end{tabular}

References

(1) Muniz, F. T.; Aurélio, M.; Miranda, R.; Morilla, C.; Santos, D.; Sasaki, J. M. The Scherrer equation and the dynamical theory of X-ray diffraction. Acta Crystallogr. 2016, 72, 385390.

(2) Plançon, A. CALCIPOW: a program for calculating the diffraction by disordered lamellar structures. J. Appl. Crystallogr. 2002, 35, 377-377.

(3) Trolard, F.; Bourrié, G.; Abdelmoula, M.; Refait, P.; Feder, F. Fougerite, a new mineral of the pyroaurite-iowaite group: Description and crystal structure. Clays Clay Miner. 2007, $55,323-334$. 